\title{
Avaliação da radiografia cefalométrica lateral como meio de diagnóstico da hipertrofia de adenoide
}

Marcelo de Castellucci e Barbosa*, Luégya Amorim Henriques Knop**, Marcus Miranda Lessa***,

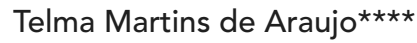

\section{Resumo}

Introdução: a hipertrofia de adenoide é uma das principais causas da respiração bucal. Entre os métodos utilizados para o diagnóstico dessa condição, os mais precisos são a endoscopia nasal e a ressonância magnética. No entanto, o método mais utilizado, em Odontologia, é a radiografia cefalométrica lateral. Objetivo: determinar a eficácia dessa radiografia no diagnóstico da hipertrofia de adenoide, pela sua comparação com a endoscopia nasal. Métodos: foram avaliados 30 indivíduos (7 a 12 anos). Todos fizeram exame de endoscopia nasal e radiografia cefalométrica lateral. Nas endoscopias, foi registrada a porcentagem de obstrução da nasofaringe e, nas radiografias, a menor dimensão anteroposterior livre da nasofaringe. Resultados: os exames se mostraram fortemente correlacionados ( $\mathrm{r}=-0,793$, p-valor $<0,01)$. Considerou-se portadores de hipertrofia severa de adenoide os pacientes que apresentaram, na endoscopia, obstrução da nasofaringe igual ou superior a $75 \%$ e, nas radiografias, o menor diâmetro anteroposterior da nasofaringe igual ou inferior a $5 \mathrm{~mm}$; o exame radiográfico teve uma sensibilidade de $75 \%$, especificidade de $86,3 \%$, valor preditivo positivo de $66,7 \%$, valor preditivo negativo de $90,4 \%$ e exatidão de $83,3 \%$. Conclusão: a radiografia cefalométrica lateral se mostrou um exame eficiente para o diagnóstico da hipertrofia de adenoide.

Palavras-chave: Hipertrofia de adenoide. Endoscopia nasal. Radiografia cefalométrica.

\section{INTRODUÇÃO}

A relação entre as funções desempenhadas pelo sistema estomatognático e o padrão de crescimento dos seus componentes é um tema bastante discutido na Odontologia. Acredita-se que a respiração é uma das funções que mais influen- ciam o crescimento facial.

Quando existe a impossibilidade, ou mesmo alguma dificuldade, de se realizar, normalmente, a respiração nasal, o indivíduo passa a respirar pela boca $^{27}$. Cassano et al. ${ }^{6}$; Kubba, Bingham ${ }^{15}$ e Tuncer et $a 1 .{ }^{30}$ defenderam que a causa mais importante

\footnotetext{
* Mestre em Odontologia pela UFBA. Especialista em Ortodontia pela PUC-MG. Professor do curso de especialização em Ortodontia da UFBA. ** Mestre em Ortodontia pela PUC-PR.

*** Doutor em Otorrinolaringologia pela FMUSP. Professor substituto de Otorrinolaringologia da UFBA.

**** Mestre e doutora em Ortodontia pela UFRJ. Professora titular de Ortodontia da UFBA.
} 
e mais frequente para a obstrução das vias aéreas superiores é a hipertrofia adenoideana. De acordo com Trotman, McNamara e Dibbets ${ }^{29}$, a via aérea superior de uma criança é estreita e a redução de apenas $1 \mathrm{~mm}$ em seu diâmetro pode diminuir o espaço aéreo efetivo em $65 \%$, produzindo uma obstrução crítica.

Quando a respiração bucal se torna habitual, diversas mudanças posturais e estruturais podem ocorrer, como postura labial incompetente, lábio superior curto, mordida aberta anterior, mordida cruzada posterior, palato atrésico e profundo, incisivos superiores projetados, além de um relacionamento oclusal de Classe II de Angle $e^{8,16,21,23}$.

No entanto, outros autores ${ }^{4,9,18}$ afirmaram ser difícil estabelecer se a obstrução das vias aéreas superiores é a responsável pela indução das alterações na morfologia craniofacial ou se essas alterações são primárias, determinadas geneticamente, facilitando a obstrução por pequenos aumentos de volume na massa adenoideana. Um dos principais aspectos questionados por esses autores é a metodologia utilizada pelos trabalhos que estabelecem a relação entre o padrão respiratório e alterações no crescimento dentofacial, principalmente no que diz respeito ao método utilizado para o diagnóstico da obstrução da nasofaringe.

Segundo alguns autores ${ }^{10,11,25}$, o exame radiográfico do crânio, em norma lateral, possibilita a medição e avaliação desse tecido linfoide, com alto grau de precisão. Para outros ${ }^{17,20,22,28}$, a radiografia cefalométrica lateral padronizada permite análise da localização, configuração e crescimento do tecido adenoideano.

Dois exames radiográficos são, comumente, utilizados para a avaliação de pacientes com suspeita de obstrução nasal: as radiografias de cavum e a cefalométrica lateral. A radiografia cefalométrica lateral é mais eficiente que a radiografia de cavum, uma vez que o posicionamento da cabeça do paciente é padronizado ${ }^{14}$.

Alguns trabalhos, no entanto, questionam a eficácia da radiografia como meio de diagnóstico da obstrução das vias aéreas superiores. Vig et al. ${ }^{31}$, em 1991, ao comparar o exame radiográfico ao de rinomanometria, chegaram à conclusão de que esse exame apresentava sensibilidade menor do que $50 \%$. Al Kindy e Obaideen ${ }^{1}$ concluíram que o exame radiográfico tem papel limitado no diagnóstico e no tratamento da hipertrofia de adenoide, gerando o desperdício de recursos e exposição desnecessária do paciente à radiação.

A ressonância magnética, de acordo com Stuck et al. ${ }^{26}$ e Welch et al. ${ }^{33}$, é uma ferramenta poderosa na determinação da anatomia nasofaringeana, por permitir exata mensuração das estruturas envolvidas, nas três dimensões. $\mathrm{O}$ alto custo, porém, inviabiliza sua utilização rotineira.

Outro método bastante eficaz, e de menor custo, é a endoscopia nasal, pois também proporciona uma imagem da nasofaringe em três dimensões, além de permitir a visualização da cor e da textura da mucosa dessa região ${ }^{13}$. $\mathrm{Hsu}^{12}$ confirmou a eficiência desse exame, ao compará-lo com a ressonância magnética e encontrar nele grande confiabilidade. Ameli et al. ${ }^{2}$ e Wang et al. ${ }^{32}$ consideraram a endoscopia nasal o melhor exame para a avaliação da nasofaringe, por ser eficiente, menos traumática e mais facilmente aceita do que outros métodos.

Segundo Kubba e Bingham ${ }^{15}$, o correto diagnóstico da hipertrofia de adenoide é essencial para a determinação do plano de tratamento adequado, que pode, inclusive, ser cirúrgico. No entanto, apesar das limitações e das controvérsias que envolvem o exame radiográfico, esse ainda é o método mais utilizado pelos cirurgiões-dentistas no diagnóstico de seus pacientes e na realização de pesquisas acerca da respiração bucal. Logo, torna-se importante uma avaliação desse método, a fim de minimizar, ao máximo, os erros de diagnóstico e de interpretação dos resultados de pesquisas científicas.

Pretendeu-se, com o presente estudo, investigar a eficácia da radiografia cefalométrica lateral no diagnóstico da hipertrofia de adenoide. 


\section{MATERIAL E MÉTODOS}

Para definição da amostra, foram selecionados pacientes da graduação e da pós-graduação em Ortodontia da Faculdade de Odontologia da Universidade Federal da Bahia e da graduação da Faculdade de Odontologia da Fundação Bahiana para o Desenvolvimento das Ciências. Dentre os pacientes das instituições citadas, participaram do estudo os 30 primeiros que necessitaram realizar a radiografia cefalométrica lateral, que não haviam sido submetidos a cirurgias otorrinolaringológicas e cujos responsáveis aceitaram que os filhos participassem da pesquisa. A amostra foi, então, composta por 30 indivíduos, sendo 14 do gênero feminino e 16 do gênero masculino, com idades variando de 7 a 12 anos.

Cada um dos indivíduos foi, então, submetido ao exame de endoscopia nasal e, no mesmo dia, à realização de uma radiografia cefalométrica lateral. As radiografias foram obtidas por um único operador, no mesmo aparelho (Rotograph Plus ${ }^{\circledR}$, Villa Systemi Medicali, Itália), utilizando de 65 a $85 \mathrm{kv}, 7 \mathrm{~mA}$ e tempo de exposição de 0,6 a 1 seg. Os filmes, da marca Kodak, foram revelados em uma processadora AT 2000 XR (Air Techniques, Inc., EUA), com tempo de processamento de, aproximadamente, 5 minutos e 30 segundos.

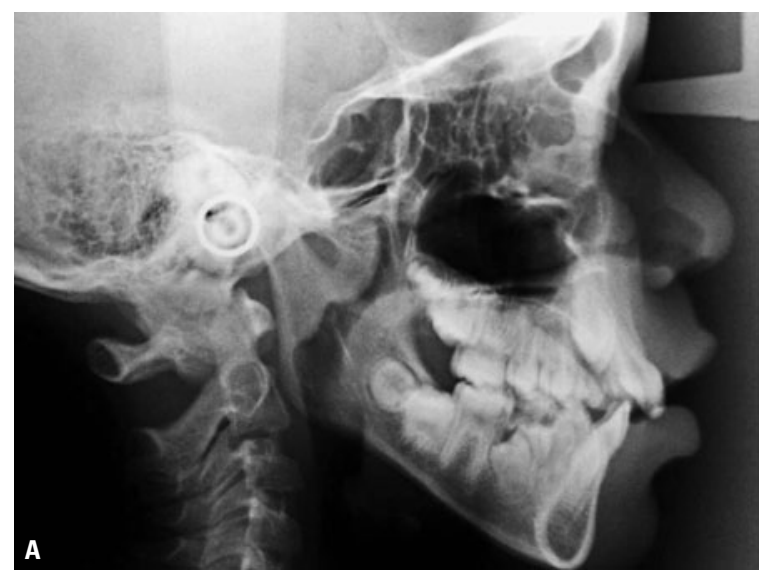

Dois exemplos das imagens radiográficas de perfil obtidas podem ser visualizados nas figuras $1 \mathrm{~A} \mathrm{e}$ 1B. Os exames de endoscopia nasal foram realizados por um único profissional, com um endoscópio flexível de 3,2mm (Machida Endoscope Co., Ltd., Tóquio, Japão) (Fig. 2). Antes da realização das endoscopias, era aplicado um anestésico tópico (xylocaína a 2\%, com adrenalina, na concentração de 1:20000) na forma de spray e, em seguida, procedia-se o exame (Fig. 3). As imagens das endoscopias (Fig. 4A, B) foram, então, gravadas em fitas de vídeo, no sistema VHS. Esse trabalho foi aprovado pelo Comitê de Ética em Pesquisa da Universidade Federal da Bahia.

Em cada radiografia, foi realizado um traçado cefalométrico manual, pelo mesmo operador, das estruturas de interesse.

A figura 5 ilustra o cefalograma utilizado nessa pesquisa. Esse foi constituído por algumas estruturas anatômicas, do crânio e da face, relatadas por Araujo $^{3}$, além de outras estruturas importantes para o estudo, como as paredes posteriores da nasofaringe e bucofaringe e o contorno posterior do palato. Nos casos de recobrimento do segmento superior da nasofaringe pela presença das vegetações adenoideanas, seu contorno anterior também foi traçado.

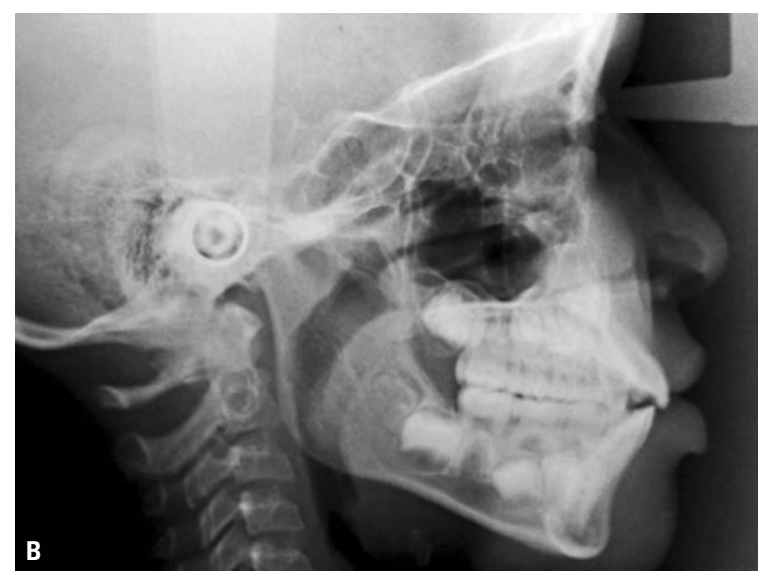

FIGURA 1 - Exemplos de imagens das radiografias cefalométricas de perfil obtidas no estudo: A) paciente portador de hipertrofia de adenoide, B) paciente sem hipertrofia de adenoide. 


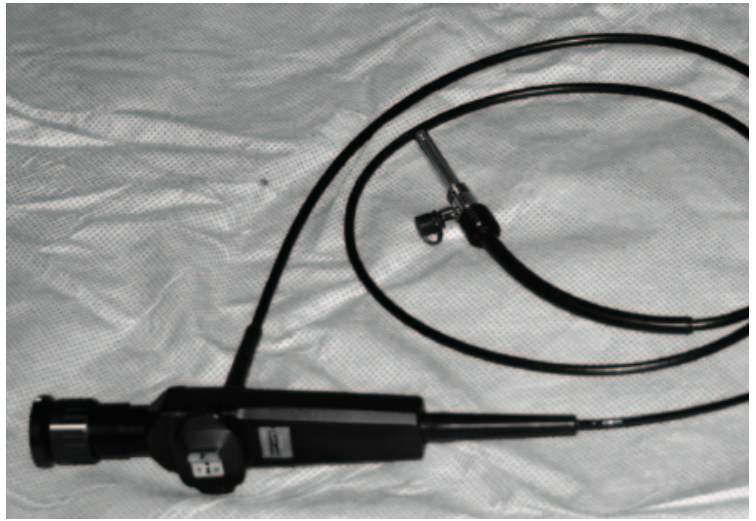

FIGURA 2 - Endoscópio flexível utilizado (3,2mm, da marca Machida).

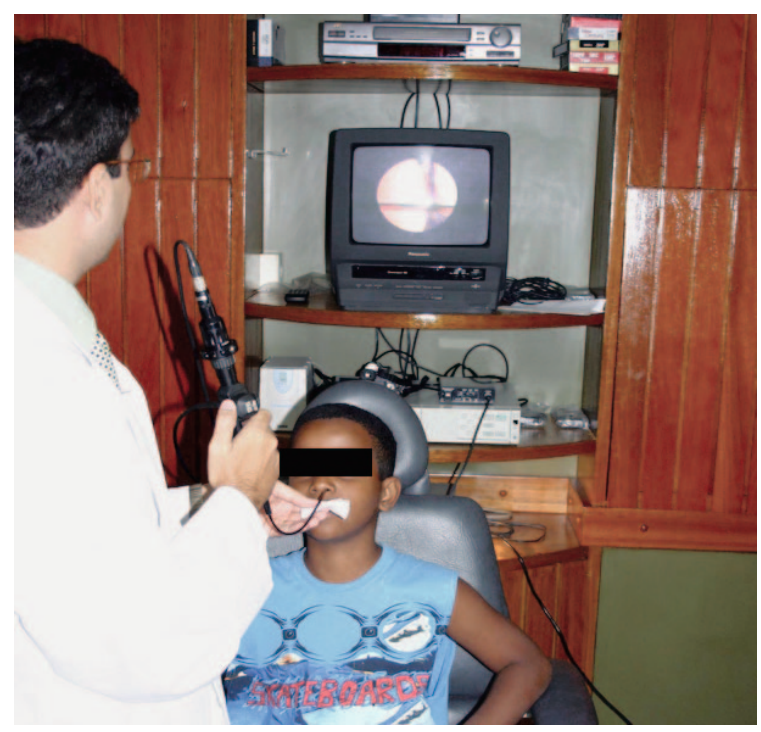

FIGURA 3 - Endoscopia nasal sendo realizada e gravada em VHS.

Sobre os traçados, determinou-se os seguintes pontos cefalométricos:

- sela (S) - centro geométrico da sela turca do osso esfenoide;

- pterigomaxilar (Ptm) - vértice da fissura pterigomaxilar, formada pela curva anterior do processo pterigoide do osso esfenoide e pelo contorno da tuberosidade retromolar da maxila;

- adenoide (Ad) - ponto de maior convexidade do contorno da adenoide;

- espinha nasal anterior (ENA) - extremidade
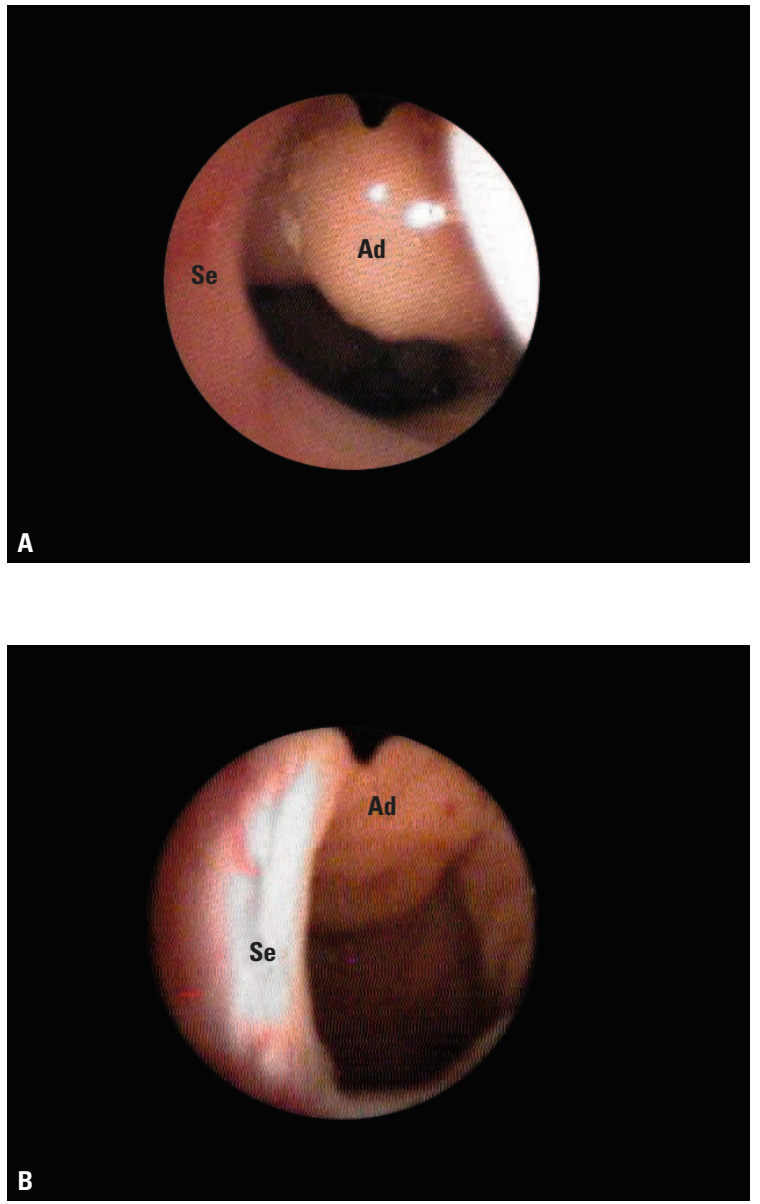

FIGURA 4 - Exemplos de imagens da nasofaringe, obtidas em endoscopias nasais, utilizando fibra flexível Machida de 3,2mm: A) coana esquerda com tecido adenoideano ocupando, aproximadamente, $80 \%$ da abertura coanal; B) coana esquerda com tecido adenoideano ocupando, aproximadamente, $10 \%$ da abertura coanal $(\mathrm{Se}=$ septo nasal; $\mathrm{Ad}=$ adenoide) .

da espinha nasal anterior;

- espinha nasal posterior (ENP) - extremidade da espinha nasal posterior;

- palato inferior $(\mathrm{Pi})$ - ponto mais inferior do palato mole.

Uma vez demarcados esses pontos, foi traçada a linha sela-pterigomaxilar (S-Ptm). Posteriormente, foi determinado o espaço livre da nasofaringe, traçando-se uma linha paralela à linha S-Ptm, passando pelo ponto Ad e indo até a parede posterior do palato mole. Da interseção dessa linha com o 
contorno posterior do palato mole, foi obtido o ponto palato mole (Pm). A linha adenoide-palato mole (Ad-Pm) corresponde ao local de maior estreitamento da nasofaringe ${ }^{5}$. O tamanho da linha Ad-Pm foi utilizado, nesse estudo, para determinar o menor diâmetro anteroposterior livre da nasofaringe. Também foram traçadas as linhas ENA-ENP e ENP-Pi e, posteriormente, medido o ângulo ENA-ENP-Pi. Esse ângulo foi determinado com o objetivo de verificar se, durante a tomada radiográfica, algum paciente teria elevado o palato mole em decorrência de algum movimento funcional, como fonação ou deglutição, o que poderia diminuir o espaço livre da nasofaringe. Para isso, foi utilizado o valor preconizado por Santos-Pinto $^{24}$ como normal para esse ângulo, que foi de 131 $\pm 5^{\circ}$. Os pontos, as linhas e o ângulo que foram utilizados podem ser observados na figura 5 .

A avaliação da endoscopia nasal foi realizada por dois avaliadores, otorrinolaringologistas previamente calibrados, em momentos diferentes. Eles não tinham conhecimento prévio do resultado do

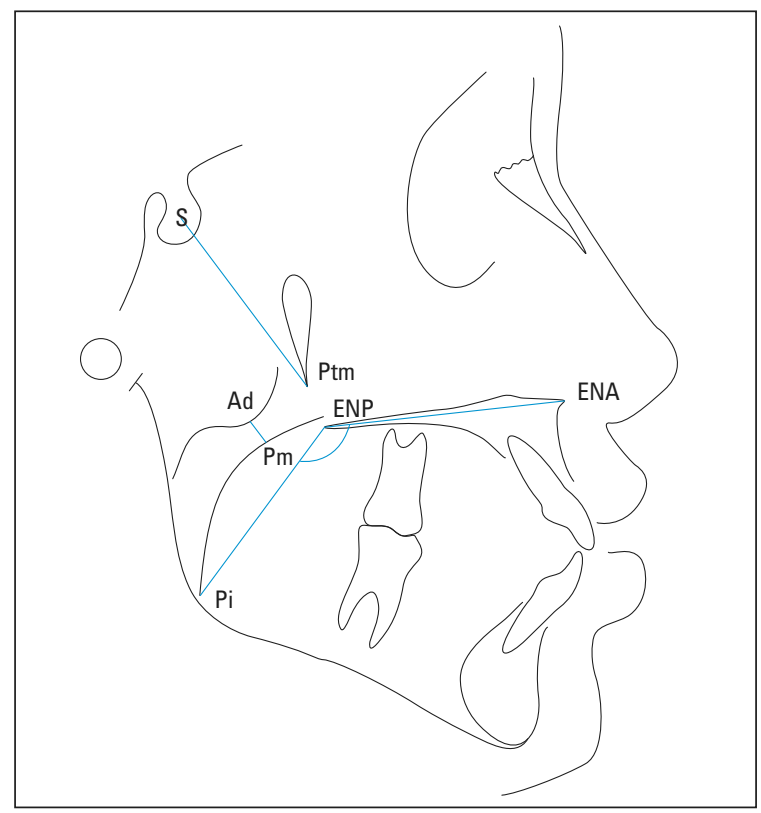

FIGURA 5 - Cefalograma representando os pontos e linhas utilizados para a determinação do espaço livre da nasofaringe. estudo radiográfico dos pacientes. Posteriormente, o grau de obstrução da nasofaringe obtido nesse exame, que foi considerado o padrão-ouro, foi registrado, sob a forma de porcentagem, e comparado aos valores obtidos na avaliação radiográfica.

Para a avaliação dos resultados dos exames, foi, inicialmente, realizado o Teste de Correlação de Pearson, a fim de verificar se existia correlação entre os valores da menor dimensão anteroposterior da nasofaringe (obtidos das radiografias) e os valores de porcentagem de obstrução da nasofaringe (obtidos das endoscopias).

Em seguida, realizaram-se os testes de validade e confiabilidade para o diagnóstico radiográfico. Foram, então, determinados a sensibilidade, a especificidade, os valores preditivos positivo e negativo, e a exatidão desse exame. Como se testou o exame radiográfico comparando-o ao endoscópico (considerado padrão-ouro), foi necessário estabelecer valores limite, para se determinar o diagnóstico positivo ou negativo para a hipertrofia da adenoide em ambos os exames. Para a avaliação endoscópica, determinou-se que, nos casos em que a obstrução da nasofaringe fosse maior ou igual a $75 \%$, o paciente seria considerado portador de hipertrofia severa da adenoide 6 . Para os resultados do exame radiográfico, os pacientes que apresentaram a menor dimensão anteroposterior livre da nasofaringe menor ou igual a $5 \mathrm{~mm}$ foram considerados portadores de hipertrofia severa da adenoide ${ }^{2,32}$.

Para todos os testes estatísticos realizados nesse trabalho, foi assumido o valor de $\mathrm{p}<0,05$ para que os resultados fossem considerados estatisticamente significantes.

\section{RESULTADOS}

A avaliação do ângulo ENA-ENP-Pi revelou que nenhum indivíduo o apresentou maior do que $131 \pm 5^{\circ}$ (valor preconizado como normal, por Santos-Pinto ${ }^{24}$, em 1984), demonstrando que não houve influência de movimentos funcionais, como deglutição ou fonação, na elevação do palato mole, com a consequente diminuição do 
espaço livre da nasofaringe.

Os valores referentes aos resultados da porcentagem de obstrução da nasofaringe pela adenoide, obtidos pela endoscopia nasal, avaliada pelo examinador 1, e os valores da menor dimensão anteroposterior da nasofaringe (obtidos pela avaliação radiográfica) estão descritos na tabela 1 .

Os valores encontrados pelos dois exames se mostraram fortemente correlacionados. O coeficiente de correlação de Pearson para as medidas encontradas foi de $-0,793$, com o p-valor $<0,01$ (Gráf. 1). Esse valor mostra uma correlação negativa entre os valores obtidos pelos dois exames, ou seja, quanto maiores os valores percentuais de obstrução da nasofaringe, obtidos pela endoscopia, menores os valores do diâmetro livre da nasofaringe, obtidos pela avaliação radiográfica.

Foram realizados os testes de validade e confiabilidade para o diagnóstico radiográfico. Em segui-

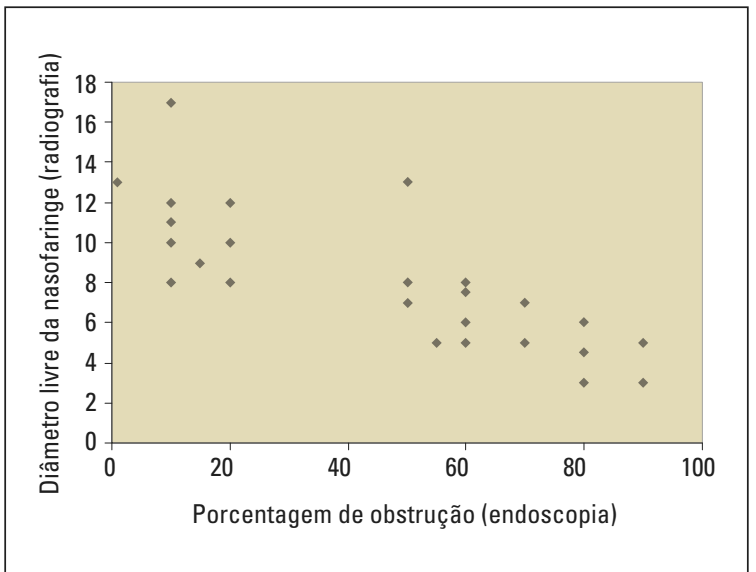

GRÁFICO 1 - Diagrama de dispersão da correlação entre os achados endoscópicos e radiográficos $(r=-0,79, p<0,01)$. da, determinou-se a sensibilidade, a especificidade, os valores preditivos positivo e negativo, e a exatidão desse exame.

Os dados utilizados para a realização dos testes de validade e confiabilidade de diagnóstico se encontram na tabela 2. Considerando a endoscopia o padrão-ouro para o diagnóstico da hipertrofia de adenoide, o exame radiográfico teve sensibilidade (proporção entre o número de diagnósticos radiográficos positivos corretos e o número total de casos positivos) de 75\%. A especificidade do exame radiográfico (proporção entre o número de diagnósticos negativos corretos e o número total de casos negativos) foi de $86,3 \%$ (Tab. 2).

O valor preditivo positivo do exame radiográfico (proporção entre o número de diagnósticos radiográficos positivos corretos e o número total de diagnósticos radiográficos positivos) foi de $66,7 \%$. $\mathrm{O}$ valor preditivo negativo (proporção entre o número de diagnósticos radiográficos negativos corretos e o número total de diagnósticos radiográficos negativos) foi de 90,4\%. Finalmente, a exatidão do exame radiográfico (proporção entre o número de diagnósticos radiográficos corretos e o número total de diagnósticos radiográficos) foi de 83,3\% (Tab. 2).

\section{DISCUSSÃO}

Alguns dos estudos que não consideram a radiografia um exame adequado para a avaliação de problemas nasofaringeanos, o fazem por não terem encontrado forte correlação entre os achados radiográficos de hipertrofia de adenoide e o padrão de respiração bucal dos pacientes avaliados ${ }^{4,9,18,31}$. Vig et al. $^{31}$, por exemplo, observaram valores baixos de sensibilidade e especificidade

TABELA 1 - Resultados da endoscopia e da radiografia cefalométrica no diagnóstico da hipertrofia de adenoide.

\begin{tabular}{|c|c|c|c|c|c|c|}
\hline variáveis & valor mínimo & valor máximo & média (d.p.) & $25 \%$ & mediana & $75 \%$ \\
\hline $\begin{array}{c}\text { endoscopia } \\
\text { (em \%) }\end{array}$ & 1 & 90 & $\begin{array}{c}48,87 \\
(30,72)\end{array}$ & 15 & 52,5 & 77,5 \\
\hline $\begin{array}{c}\mathbf{R X} \\
(\mathrm{em} \mathrm{mm})\end{array}$ & 3 & 17 & $\begin{array}{c}7,78 \\
(3,37)\end{array}$ & 5 & 7,75 & 9,75 \\
\hline
\end{tabular}


TABELA 2 - Distribuição dos pacientes, segundo os resultados dos exames endoscópicos e radiográficos.

\begin{tabular}{cccc} 
RADIOGRAFIA & \multicolumn{2}{c}{ ENDOSCOPIA } & TOTAL \\
& positivo & negativo & \\
\hline positivo & 6 & 3 & 9 \\
negativo & 2 & 19 & 21 \\
total & 8 & 22 & 30 \\
\hline
\end{tabular}

para a radiografia no diagnóstico de respiração bucal, comparando seus resultados aos do exame de rinomanometria, que avalia a resistência nasal. No entanto, o exame radiográfico não pode ser utilizado isoladamente para dar o diagnóstico de respiração bucal. Ele deve ser indicado para a avaliação de características anatômicas da nasofaringe como, por exemplo, do volume adenoideano e, a partir daí, nos casos de detecção de anormalidades, o paciente deve ser encaminhado para avaliações mais completas. Não foi a intenção do presente trabalho sugerir a radiografia cefalométrica como principal meio de diagnóstico para a respiração bucal, e sim avaliar sua importância no diagnóstico de uma das condições mais frequentemente associadas a esses pacientes, que é a hipertrofia de adenoide ${ }^{6,15,30}$.

Para alguns autores ${ }^{2,12,13,32}$, a endoscopia nasal é um exame de excelência no diagnóstico de problemas localizados na nasofaringe, por permitir a visualização praticamente direta da região, em três dimensões, além de possibilitar a observação da cor e da textura da mucosa envolvida. Portanto, avaliar a eficiência da radiografia cefalométrica lateral no diagnóstico da hipertrofia de adenoide, pela comparação de seus resultados aos de endoscopias nasais, parece ser um método eficaz.

Os achados do presente trabalho, que indicam a eficiência do exame radiográfico no diagnóstico da hipertrofia de adenoide, estão de acordo com os trabalhos de Ikino et al. ${ }^{14}$; McNamara ${ }^{17}$; Poole, Engel, Chaconas ${ }^{20}$; Pruzansky ${ }^{22}$ e Subtelny ${ }^{28}$, mas contradizem os achados de Al Kindy, Obaideen ${ }^{1}$;
Ameli et al. ${ }^{2}$; Vig et al. ${ }^{31}$ e Wang et al. ${ }^{32}$

Nem todos os trabalhos que consideram a radiografia um exame de pouca utilidade para o diagnóstico de problemas nasofaringeanos utilizaram a radiografia cefalométrica lateral. A falta de padronização das tomadas radiográficas utilizadas pode ter influenciado nos resultados negativos desse exame. Como foi mostrado por Ikino et al. ${ }^{14}$, a radiografia de cavum, bastante utilizada entre os otorrinolaringologistas, apresenta, no diagnóstico da hipertrofia de adenoide, eficiência bem inferior à da radiografia cefalométrica. O fato de, na presente pesquisa, ter sido utilizada apenas a radiografia cefalométrica lateral, com a posição da cabeça do paciente padronizada pelo cefalostato, pode ter influenciado, positivamente, nos resultados de eficiência desse exame que, mesmo em duas dimensões, foi capaz de evidenciar a hipertrofia de adenoide. No entanto, apesar dos resultados positivos encontrados nesse trabalho, não se pode dizer que o exame radiográfico é o melhor método para o diagnóstico da hipertrofia de adenoide. Ao ser comparado à endoscopia, a sensibilidade do exame radiográfico, ou seja, a probabilidade de o exame dar resultado positivo quando o paciente apresenta a condição, foi de $75 \%$; e o valor preditivo positivo, ou seja, a probabilidade de um exame que teve resultado positivo pertencer a um indivíduo que é portador da condição, foi de $66,7 \%$. Os dois, principalmente o primeiro, são valores altos ${ }^{7,19}$, que recomendam a utilização desse exame para a finalidade em questão. Contudo, o exame radiográfico não pode substituir, em todas as situações, os outros exames de imagem, reconhecidamente mais eficientes. A radiografia é, sem dúvida, mais eficiente que exames como a palpação digital e a inspeção visual direta, mas a endoscopia nasal e a ressonância magnética continuam sendo exames mais completos e eficazes. No entanto, esses nem sempre são acessíveis para os pacientes, seja por limitações financeiras ou por falta de equipamentos disponíveis.

Esse aspecto se torna ainda mais importante quando se trata de pacientes que irão se submeter 
a um tratamento ortodôntico e que, por isso, farão a radiografia cefalométrica lateral com finalidade de diagnóstico, independentemente de sua condição respiratória. Para esses pacientes, a utilidade desse exame é ainda maior, pois pode evitar os custos financeiro e biológico da realização desnecessária de outro exame.

A determinação de um plano de tratamento cirúrgico envolve uma série de outros fatores, como dimensão livre remanescente da nasofaringe, idade do paciente, condições sistêmicas, entre outros. Portanto, naqueles casos nos quais a radiografia cefalométrica indica a hipertrofia de adenoide, o paciente deve ser encaminhado para fazer outros exames, a fim de que o tratamento adequado seja recomendado. Portanto, é mais importante que os resultados negativos para essa condição sejam fiéis do que, propriamente, os positivos, já que um resultado radiográfico negativo para hipertrofia de adenoide pode fazer com que o paciente não seja referido para o tratamento dessa condição, caso o mesmo não apresente outros sinais ou sintomas de distúrbios respiratórios. $\mathrm{E}$ os testes que avaliam esse aspecto foram ainda mais positivos ${ }^{7}, 19$.
A especificidade do exame radiográfico, ou seja, a probabilidade de dar resultado negativo quando o paciente não apresenta a condição, foi de $86,3 \%$. E o valor preditivo negativo, que expressa a probabilidade de o indivíduo não apresentar o problema quando o resultado do exame é negativo, foi de 90,4\%. Logo, a radiografia cefalométrica lateral se mostrou um bom exame para o diagnóstico da hipertrofia de adenoide, principalmente para descartar a existência do problema, o que, como já foi mencionado, é ainda mais importante.

\section{CONCLUSÃO}

A radiografia cefalométrica lateral se mostrou um exame eficiente, com sensibilidade, especificidade e valores preditivos positivo e negativo altos para o diagnóstico da hipertrofia de adenoide. Isso foi comprovado pela forte correlação entre os seus resultados e os da endoscopia nasal, que é considerada o exame padrão-ouro para o diagnóstico dessa condição.

\title{
Evaluation of lateral cephalometric radiography as a mean of diagnosing adenoids hypertrophy
}

\begin{abstract}
Introduction: One of the most usual causes of mouth breathing is adenoids hypertrophy with reduction of the nasopharyngeal space. The most precise diagnostic methods are magnetic resonance and nasal endoscopy, because they make possible a three dimension image of the nasopharynx. However, in Dentistry, cephalometric radiography is the method used in the majority of cases. That is why it is so important the evaluation of the efficacy of this diagnostic method. Aim: The aim of this paper is to determine the efficacy of the lateral cephalometric radiography in diagnosing adenoids hypertrophy, comparing this method to the nasal endoscopy. Methods: Thirty patients (7 to 12 years), with no history of otolaryngological surgery, were evaluated. All of them were submitted to a nasal endoscopy and a lateral cephalometric radiography. In the endoscopic exams it was registered the percentage of nasopharyngeal obstruction and in the radiographic exams it was registered the minor nasopharyngeal dimension. Results: The results of the exams showed a strong correlation with each other $(r=-0.793$, $p$-value $<0.01)$. After that, reliability tests to the radiographic diagnose were performed, assuming that $75 \%$ (endoscopic exams) and $5 \mathrm{~mm}$ (radiographic exams) were the limit values to the determination of the diagnose of severe adenoids hypertrophy. The radiographic exam showed a sensibility of $75 \%$, specificity of $86.3 \%$, positive predictive value of $66.7 \%$, negative predictive value of $90.4 \%$ and an exactness of $83.3 \%$. Therefore, lateral cephalometric radiography is an efficient method of adenoids hypertrophy diagnose. It was proved by the strong correlation of its results with the results of the nasal endoscopy, that is considered a method of excellence for diagnosing this condition.
\end{abstract}

Keywords: Adenoids hypertrophy. Nasal endoscopy. Cephalometric radiography. 


\section{REFERÊNCIAS}

1. AL KINDY, S. A.; OBAIDEEN, A. O. The value of radiological examination in the management of adenoidal hypertrophy in a pediatric population. Saudi Med. J., Riyadh, v. 24, no. 5, p. 504-506, May 2003.

2. AMELI, F. et al. Nasal endoscopy in asthmatic children: clinical role in the diagnosis of rhinosinusitis. Rhinology, Leiden, v. 42, no. 1, p. 15-18, Mar. 2004

3. ARAUJO, T. M. Cefalometria, conceitos e análises. 1983. 304 f. Dissertação (Mestrado)-Faculdade de Odontologia, Universidade Federal do Rio de Janeiro, Rio de Janeiro, 1983.

4. BERKINSHAW, E. R.; SPALDING, P. M.; VIG, P. S. The effect of methodology on the determination of nasal resistance. Am. J. Orthod. Dentofacial Orthop., St. Louis, v. 92, no. 4, p. 329-335, Oct. 1987.

5. BITTENCOURT, M. A. V. Dimensão da nasofaringe em indivíduos portadores da maloclusão de Classe II. $2001.68 \mathrm{f}$. Trabalho de Conclusão de Curso (Especialização)-Faculdade de Odontologia, Universidade Federal da Bahia, Salvador, 2001.

6. CASSANO, P. et al. Adenoid tissue rhinopharyngeal obstruction grading based on fiberendoscopy findings: a novel approach to therapeutic management. Int. J. Pediatr. Otorhinolaryngol, Limerick, v. 67, no. 12, p. 1303-1309, Dec. 2003.

7. ESTRELA, C. Princípios de bioestatística. In: Metodologia científica: ensino e pesquisa em Odontologia. São Paulo: Artes Médicas, 2001. cap. 14, p. 277-305.

8. FERES, M. A. N.; MATSUMOTO, M. A. N.; ENOKI, C. Nasopharyngeal dimensions in hyperdivergent facial skeletal type. In: INTERNATIONAL SYMPOSIUM ON DENTOFACIAL DEVELOPMENT AND FUNCTION, 10., 2004, Costa do Sauípe. Abstracts Book... Costa do Sauípe: [s.n.], 2004. p. 73.

9. FIELDS, H. W.; WARREN, D. W.; BLACK, K. et al. Relationship between vertical dentofacial morphology and respiration in adolescents. Am. J. Orthod. Dentofacial Orthop., St. Louis, v. 99, no. 2, p. 147-154, Feb. 1991.

10. HIBBERT, J.; WHITEHOUSE, G. H. The assessment of adenoidal size by radiological means. Clin. Otolaryngol. Allied Sci., Oxford, v. 3, no. 1, p. 43-47, Feb. 1978.

11. HOLMBERG, H.; LINDER-ARONSON, S. Cephalometric radiographs as a mean of evaluating the capacity of the nasal and nasopharyngeal airway. Am. J. Orthod., St. Louis, v. 76, no. 5, p. 479-490, Nov. 1979.

12. HSU, P. P. A new method of evaluation of upper airway in patients with obstructive sleep apnoea: computer-assisted quantitative videoendoscopic analysis. Ann. Acad. Med. Singap., Singapore, v. 31, no. 3, p. 393-398, May 2002.

13. IANNI FILHO, D. et al. A comparison of nasopharyngeal endoscopy and lateral cephalometric radiography in the diagnosis of nasopharyngeal airway obstruction. Am. J. Orthod. Dentofacial Orthop., St. Louis, v. 120, no. 4, p. 348-352, Oct. 2001.

14. IKINO, C. M. Y. et al. Telerradiografia lateral de crânio e radiografia de cavum: estudo comparativo em crianças com obstrução nasal. Rev. Bras. Otorrinolaringol., São Paulo, v. 66, n. 6, p. 592-596, nov./dez. 2000.

15. KUBBA, H.; BINGHAM, B. J. Endoscopy in the assessment of children with nasal obstruction. J. Laryngol. Otol., Cambridge, v. 115, no. 5, p. 380-384, May 2001.

16. McNAMARA, J. A. Influence of respiratory pattern of craniofacial growth. Angle Orthod., Appleton, v. 51, no. 4, p. 269-300, Oct. 1981.

17. McNAMARA, J. A. A method of cephalometric evaluation. Am. J. Orthod., St. Louis, v. 86, no. 6, p. 449-469, Dec. 1984.

18. O'RYAN, F. S.; GALLAGHER, D. M.; LaBANC, J. P. et al. The relation between nasorespiratory function and dentofacial morphology: a review. Am. J. Orthod., St. Louis, v. 82, no. 5, p. 403-410, Nov. 1982.

19. PEREIRA, M. G. Validade de uma investigação. In: Epidemiologia: teoria e prática. Rio de Janeiro: Guanabara Koogan, 2002. cap. 15, p. 308-336.
20. POOLE, M. N.; ENGEL, G. A.; CHACONAS, S. J. Nasopharyngeal cephalometrics. Oral Surg. Oral Med. Oral Pathol., St. Louis, v. 49, no. 3, p. 266-271, Mar. 1980.

21. PRESTON, C. B. Chronic nasal obstruction and malocclusion. J. Dent. Assoc. S. Afr., Cape Town, v. 36, no. 11, p. 759-763, Nov. 1981.

22. PRUZANSKY, S. Roentgencephalometric study of tonsils and adenoids in normal and pathologic states. Ann. Otol. Rhinol. Laryngol., St. Louis, v. 84, no. 2, p. 55-62, Mar./Apr. 1975. Supplement 19

23. RUBIN, R. M. Mode of respiration and facial growth. Am. J. Orthod., St. Louis, v. 78, no. 5, p. 504-510, Nov. 1980.

24. SANTOS-PINTO, A. Alterações nasofaringeanas e craniofaciais em pacientes com adenóide hipertrófica: estudo cefalométrico. 1984. Dissertação (Mestrado)- Faculdade de Odontologia, Universidade Federal do Rio de Janeiro, Rio de Janeiro, 1984.

25. SORENSEN, H.; SOLOW, B.; GREVE, E. Assessment of nasopharyngeal airway: a rhinomanometric and radiographic study in children with adenoids. Acta Otolaryngol., Oslo, v. 89, no. 3/4, p. 227-232, Mar./Apr. 1980.

26. STUCK, B. A. et al. Evaluating the upper airway with standardized magnetic resonance imaging. Laryngoscope, Philadelphia, v. 112, no. 3, p. 552-558, Mar. 2002.

27. SUBTELNY, J. D. The significance of adenoid tissue in Orthodontia. Angle Orthod., Appleton, v. 24, no. 2, p. 59-69, Apr. 1954

28. SUBTELNY, J. D. Effect of diseases of tonsils and adenoids on dentofacial morphology. Ann. Otol. Rhinol. Laryngol., St. Louis, v. 84, p. 50-54, Mar./Apr. 1975. Supplement 19.

29. TROTMAN, C. A.; McNAMARA, J. A.; DIBBETS, J. M. et al. Association of lip posture and the dimensions of the tonsils and sagittal airway with facial morphology. Angle Orthod., Appleton, v. 67, no. 6, p. 425-432, 1997.

30. TUNCER, U. et al. Chronic rhinosinusitis and adenoid hypertrophy in children. Am. J. Otolaryngol., Cherry Hill, v. 25, no. 1, p. 5-10, Jan./Feb. 2004.

31. VIG, P. et al. Sensitivity and specificity of diagnostic tests for impaired nasal respiration. Am. J. Orthod. Dentofacial Orthop., St. Louis, v. 99, no. 4, p. 354-360, Apr. 1991.

32. WANG, D. Y. et al. Assessment of adenoid size in children by fiber-optic examination. Clin. Otolaryngol., Oxford, v. 22, no. 2, p. 172-177, Jan. 1997

33. WELCH, K. C. et al. A novel volumetric magnetic resonance imaging paradigm to study upper airway anatomy. Sleep, Winchester, v. 25, no. 5, p. 532-542, Aug. 2002.
Endereço para correspondência Marcelo de Castellucci e Barbosa Rua Araújo Pinho, 62, $7^{\circ}$ andar, Canela CEP: 40.110-150 - Salvador / BA E-mail: mcastellucci@uol.com.br 